\title{
Gene Ontology
}

National Cancer Institute

\section{Source}

National Cancer Institute. Gene Ontology. NCI Thesaurus. Code C43520.

The Gene Ontology (GO) project is a collaborative effort to address the need for consistent descriptions of gene products in different databases. The goal of the Gene Ontology project is to produce a controlled vocabulary that can be applied to all organisms even as knowledge of gene and protein roles in cells is accumulating and chang ing. GO provides three structured networks of defined terms, molecular function, biological process, and cellular component, to describe gene product attributes. 